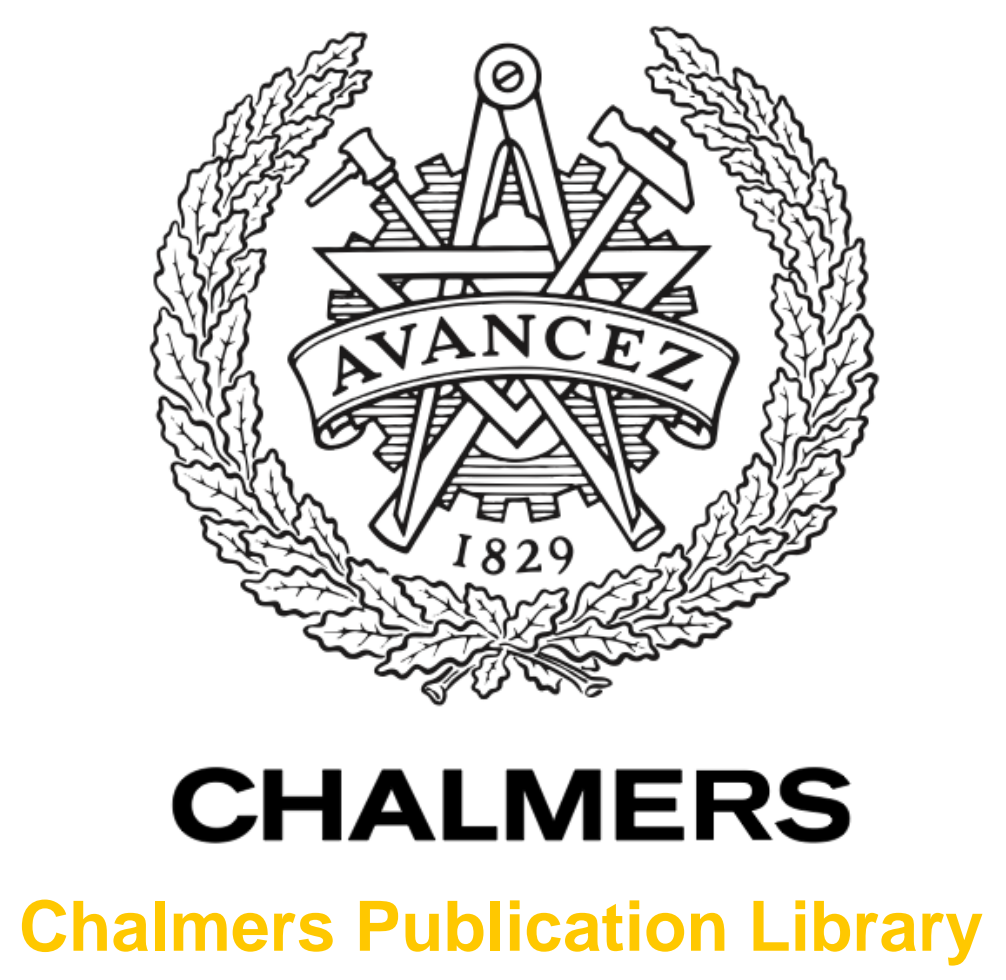

Robust power allocation for OFDM wireless network localization

This document has been downloaded from Chalmers Publication Library (CPL). It is the author's version of a work that was accepted for publication in:

IEEE International Conference on Communication Workshop, ICCW 2015 (ISSN: 2164-7038)

Citation for the published paper:

Shahmansoori, A. ; Seco-Granados, G. ; Wymeersch, H. (2015) "Robust power allocation for OFDM wireless network localization". IEEE International Conference on Communication Workshop, ICCW 2015 pp. 718-723.

http://dx.doi.org/10.1109/ICCW.2015.7247266

Downloaded from: http://publications.lib.chalmers.se/publication/227702

Notice: Changes introduced as a result of publishing processes such as copy-editing and formatting may not be reflected in this document. For a definitive version of this work, please refer to the published source. Please note that access to the published version might require a subscription.

Chalmers Publication Library (CPL) offers the possibility of retrieving research publications produced at Chalmers University of Technology. It covers all types of publications: articles, dissertations, licentiate theses, masters theses, conference papers, reports etc. Since 2006 it is the official tool for Chalmers official publication statistics. To ensure that Chalmers research results are disseminated as widely as possible, an Open Access Policy has been adopted.

The CPL service is administrated and maintained by Chalmers Library. 


\title{
Robust Power Allocation for OFDM Wireless Network Localization
}

\author{
Arash Shahmansoori*, Gonzalo Seco-Granados*, and Henk Wymeersch ${ }^{\dagger}$ \\ *Universitat Autònoma de Barcelona, Spain, Email: \{arash.shahmansoori, gonzalo.seco\}@uab.cat \\ ${ }^{\dagger}$ Chalmers University of Technology, Sweden, Email: henkw@chalmers.se
}

\begin{abstract}
Reliable and accurate localization of users is critical for many applications in wireless networks. In range-based localization, the position of a node (agent) can be estimated using distance measurements to nodes with known positions (anchors). Optimal power allocation of the anchors reduces positioning error and improves network lifetime and throughput. We formulate and solve a robust power allocation optimization problem with a constraint on the localization accuracy for multicast and unicast orthogonal frequency division multiplexing signals. The localization accuracy is expressed in terms of the expected squared position error bound, accounting for uncertainties in the wireless channel as well as in the agents' positions. Simulation results show that robust power allocation improves localization accuracy compared to non-robust power allocation, with only a limited power penalty.
\end{abstract}

\section{INTRODUCTION}

High-accuracy localization is of critical importance in many location-based applications and services, e.g., cellular positioning, search and rescue tasks, blue-force tracking, communication, and military systems [1], [2]. Wireless network localization (WNL) refers to the process of finding the positions of users (agents) using measurements (e.g., range measurements) to nodes with known positions (anchors). The transmission power of the nodes plays an important role in WNL, not only in terms of lifetime and throughput, but also in positioning accuracy [3]. Therefore, an optimal power allocation is important for reducing power consumption and increasing positioning accuracy.

Several power allocation methods have been presented for single-carrier transmission in synchronized networks [4], [5]. These methods include the positioning accuracy as either an objective or a constraint, using fundamental performance limits. In [6], [7], the fundamental limits of wideband localization have been derived in terms of squared position error bound (SPEB) and directional position error bound (DPEB) for the case of single-carrier signals. To account for the uncertainties in the network parameters, a robust power allocation has been proposed in [8], using semidefinite programming (SDP) and second-order cone programming (SOCP) forms, minimizing SPEB and maximum DPEB (mDPEB) subject to a total power constraint. In [4], [5], [8], it is assumed that when an anchor sends a signal to a particular agent, other agents cannot listen (unicast assumption). However, for a synchronous network, this leads to suboptimal solutions in terms of total required power for the anchors. Moreover, unlike in [4]-[8], current and emerging communications standards generally employ multicarrier signals, in particular orthogonal frequency division multiplexing (OFDM). Multicarrier transmissions are beneficial when the data rates increase and wider bandwidths are needed. Also, they are less susceptible to interference compared to single-carrier transmission as interference may only affect a small number of subcarriers. Hence, it is important to understand the power allocation problem for OFDM WNL.

In this paper, we present a framework for robust power allocation in an OFDM WNL with uncertainties on the network parameters, which include the channel coefficients and the positions of the agents. Specifically, we use the SPEB as the fundamental limit of the localization accuracy. Our main contributions are summarized as follows.

- We derive the SPEB for OFDM signals in a synchronous network, for the cases of (i) deterministic unknown agent positions and channels; (ii) a collection of agent positions and channels drawn from suitable distributions.

- We develop a method to obtain subcarrier power allocations that minimize the total transmitted power, subject to a constraint on the average SPEB, for both unicast and multicast transmissions.

Notation: $\operatorname{diag}\left\{\mathbf{X}_{m}\right\}_{m \in \mathcal{M}}$ denotes the block-diagonal matrix with the $m$ th block equal to $\mathbf{X}_{m}$ and $m$ in the set of indices $\mathcal{M} ; \operatorname{diag}\left\{x_{m}\right\}_{m=0}^{M-1}$ denotes an $M \times M$ diagonal matrix; $\mathbf{I}_{N}$ is an $N \times N$ identity matrix; $\|$.$\| denotes the Euclidean norm;$ $\otimes$ denotes the Kronecker product; $\mathbf{X} \succeq \mathbf{Y}$ means that the matrix $\mathbf{X}-\mathbf{Y}$ is positive semi-definite (PSD); $\mathbf{X} \succ \mathbf{Y}$ means that the matrix $\mathbf{X}-\mathbf{Y}$ is positive definite; $\mathbf{x} \succeq \mathbf{y}$ and $\mathbf{x} \succ \mathbf{y}$ denote all the elements of $\mathbf{x}-\mathbf{y}$ are nonnegative and positive, respectively; $\mathbf{0}$ denotes a column vector with all 0 's; $\Re\{$. and $\Im\{$.$\} denote the real and imaginary part of the argument,$ respectively.

\section{System MODEL}

Consider a wireless network with $N_{b}$ anchors with known positions and $N_{a}$ agents with unknown positions. The sets of agents and anchors are denoted by $\mathcal{N}_{a}=\left\{1, \ldots, N_{a}\right\}$ and $\mathcal{N}_{b}=\left\{N_{a}+1, \ldots, N_{a}+N_{b}\right\}$. The two-dimensional positions of $k$ th agent and $j$ th anchor are denoted by $\mathbf{q}_{k}=\left[\begin{array}{ll}x_{k} & y_{k}\end{array}\right]^{\mathrm{T}}$ for $k \in \mathcal{N}_{a}$ and $\mathbf{q}_{j}=\left[\begin{array}{ll}x_{j} & y_{j}\end{array}\right]^{\mathrm{T}}$ for $j \in \mathcal{N}_{b}$. Anchors may be elements of the fixed infrastructure like cellular base stations, whereas agents may be mobile users. We assume all nodes are perfectly synchronized and use OFDM transmissions from anchors to agents to localize the agents. We consider both multicast and unicast communication. 
Multicast operation: Each anchor $j$ sends an OFDM signal with $N$ subcarriers, which is received by all agents. We denote as $\mathbf{r}_{k, j}$ the $N \times 1$ vector representing the received signal by agent $k$ due to the transmission of anchor $j$, after cyclic prefix removal and transformation to the frequency domain. The vector $\mathbf{r}_{k, j}$ can be expressed as [9]

$$
\mathbf{r}_{k, j}=\boldsymbol{\Gamma}\left(t_{k, j}\right) \mathbf{B}_{j} \mathbf{F}_{L_{k, j}} \mathbf{h}_{k, j}+\mathbf{w}_{k, j}
$$

in which $\mathbf{B}_{j}=\operatorname{diag}\left\{B_{j}[n]\right\}_{n=0}^{N-1}$ is an $N \times N$ diagonal matrix representing the signals transmitted by anchor $j$ on each of the subcarriers, $\mathbf{F}_{L_{k, j}}$ represents the first $L_{k, j}$ columns of the $N \times N$ discrete Fourier transform (DFT) matrix with $L_{k, j}$ being the number of channel taps between nodes $j$ and $k, \mathbf{h}_{k, j}=\left[\begin{array}{lll}h_{k, j}^{(1)} & \ldots & h_{k, j}^{\left(L_{k, j}\right)}\end{array}\right]^{\mathrm{T}}$ is the sampled channel impulse response between anchor $j$ and agent $k$ with the real and imaginary parts defined as $\mathbf{h}_{\mathrm{R}, k, j}$ and $\mathbf{h}_{\mathrm{I}, k, j}$, respectively, $\boldsymbol{\Gamma}\left(t_{k, j}\right)=\operatorname{diag}\left\{\exp \left(-j 2 \pi n t_{k, j} / T\right)\right\}_{n=0}^{N-1}$ where $t_{k, j}$ is the delay of the first path and is given by $t_{k, j}=\left\|\mathbf{q}_{k}-\mathbf{q}_{j}\right\| / c$ with $c$ representing the speed of light, $T$ is the OFDM symbol duration, and $\mathbf{w}_{k, j}$ is an $N \times 1$ noise vector distributed as $\mathcal{C N}\left(\mathbf{0}, \sigma_{w}^{2} \mathbf{I}_{N}\right)$. We introduce $\mathbf{r}$ as the vector representation of all the received waveforms, given by $\mathbf{r}=\left[\begin{array}{llll}\mathbf{r}_{1}^{\mathrm{T}} & \ldots & \mathbf{r}_{N_{a}}^{\mathrm{T}}\end{array}\right]^{\mathrm{T}}$ with $\mathbf{r}_{k}=\left[\begin{array}{llll}\mathbf{r}_{k, N_{a}+1}^{\mathrm{T}} & \ldots & \mathbf{r}_{k, N_{a}+N_{b}}^{\mathrm{T}}\end{array}\right]^{\mathrm{T}}$.

Unicast operation: Each anchor $j$ will send different OFDM signals to each agent. In terms of the model, the only difference is that in (1) we replace $\mathbf{B}_{j}$ by $\mathbf{B}_{k, j}$.

\section{Goal}

Our goal is to minimize the total transmission power (related to $\mathbf{P}_{j}=\mathbf{B}_{j}^{\mathrm{H}} \mathbf{B}_{j}$ for multicast and to $\mathbf{P}_{k, j}=\mathbf{B}_{k, j}^{\mathrm{H}} \mathbf{B}_{k, j}$ for unicast) to obtain a certain positioning accuracy in the presence of uncertainties in the channels and the agents' positions.

For notational convenience, we introduce $\mathbf{p}_{j}$ as the $N \times 1$ vector taken from the main diagonal of $\mathbf{P}_{j}$, as well as $\mathbf{p}_{k, j}$ as the $N \times 1$ vector taken from the main diagonal of $\mathbf{P}_{k, j}$. We further introduce $\mathbf{p}=\left[\mathbf{p}_{1}^{\mathrm{T}}, \ldots, \mathbf{p}_{N_{b}}^{\mathrm{T}}\right]^{\mathrm{T}}$ and $\mathbf{p}_{k,:}=\left[\mathbf{p}_{k, 1}^{\mathrm{T}}, \ldots, \mathbf{p}_{k, N_{b}}^{\mathrm{T}}\right]^{\mathrm{T}}$.

\section{SQuAREd Position ERror Bound}

In this section, we derive the Fisher information matrix (FIM) of the agents' positions with channel coefficients treated as deterministic unknown parameters. Then, the SPEB is determined for two cases: for a particular value of the agents' positions and channel coefficients, and for a set of agents' positions and channel coefficients. The latter SPEB will later be used for a robust power allocation. Our derivation will consider the multicast problem, but we will revisit the distinction between unicast and multicast again in Section IV.

\section{A. Derivation of the FIM}

Let $\hat{\eta}$ be an unbiased estimator of

$$
\boldsymbol{\eta}=\left[\begin{array}{llllll}
\mathbf{q}_{1}^{\mathrm{T}} & \ldots & \mathbf{q}_{N_{a}}^{\mathrm{T}} & \boldsymbol{\theta}_{1}^{\mathrm{T}} & \ldots & \boldsymbol{\theta}_{N_{a}}^{\mathrm{T}}
\end{array}\right]^{\mathrm{T}},
$$

where $\boldsymbol{\theta}_{k}=\left[\mathbf{k}_{k, N_{a}+1}^{\mathrm{T}}, \ldots, \mathbf{k}_{k, N_{a}+N_{b}}^{\mathrm{T}}\right]^{\mathrm{T}}$, with $\mathbf{k}_{k, j}=$ $\left[\begin{array}{ll}\mathbf{h}_{R, k, j}^{\mathrm{T}} & \mathbf{h}_{I, k, j}^{\mathrm{T}}\end{array}\right]^{\mathrm{T}}$. The mean squared error (MSE) of $\hat{\boldsymbol{\eta}}-\boldsymbol{\eta}$ is bounded as [10]

$$
\mathbb{E}_{\mathbf{r} \mid \boldsymbol{\eta}}\left[(\hat{\boldsymbol{\eta}}-\boldsymbol{\eta})(\hat{\boldsymbol{\eta}}-\boldsymbol{\eta})^{\mathrm{T}}\right] \succeq \mathbf{J}_{\boldsymbol{\eta}}^{-1},
$$

where $\mathbb{E}_{\mathbf{r} \mid \boldsymbol{\eta}}[$.$] denotes the expectation over the noise distribu-$ tion, $\mathbf{J}_{\boldsymbol{\eta}}$ is the $2\left(\bar{L}+N_{a}\right) \times 2\left(\bar{L}+N_{a}\right)$ FIM with $\bar{L}$ defined as $\bar{L}=\sum_{k=1}^{N_{a}} \sum_{j=N_{a}+1}^{N_{a}+N_{b}} L_{k, j}$, and

$$
\mathbf{J}_{\boldsymbol{\eta}}=\mathbb{E}_{\mathbf{r} \mid \boldsymbol{\eta}}\left[-\frac{\partial^{2} \ln f(\mathbf{r} ; \boldsymbol{\eta})}{\partial \boldsymbol{\eta} \partial \boldsymbol{\eta}^{T}}\right] .
$$

The FIM has the following form

$$
\mathbf{J}_{\boldsymbol{\eta}}=\left[\begin{array}{cccc}
\mathbf{\Phi}(\mathbf{q}, \mathbf{q}) & \mathbf{\Phi}\left(\mathbf{q}, \boldsymbol{\theta}_{1}\right) & \ldots & \boldsymbol{\Phi}\left(\mathbf{q}, \boldsymbol{\theta}_{N_{a}}\right) \\
\mathbf{\Phi}\left(\boldsymbol{\theta}_{1}, \mathbf{q}\right) & \mathbf{\Phi}\left(\boldsymbol{\theta}_{1}, \boldsymbol{\theta}_{1}\right) & \ldots & \boldsymbol{\Phi}\left(\boldsymbol{\theta}_{1}, \boldsymbol{\theta}_{N_{a}}\right) \\
\vdots & & \ddots & \vdots \\
\mathbf{\Phi}\left(\boldsymbol{\theta}_{N_{a}}, \mathbf{q}\right) & \boldsymbol{\Phi}\left(\boldsymbol{\theta}_{N_{a}}, \boldsymbol{\theta}_{1}\right) & \ldots & \boldsymbol{\Phi}\left(\boldsymbol{\theta}_{N_{a}}, \boldsymbol{\theta}_{N_{a}}\right)
\end{array}\right]
$$

in which $\mathbf{q}=\left[\mathbf{q}_{1}^{\mathrm{T}}, \ldots, \mathbf{q}_{N_{a}}^{\mathrm{T}}\right]^{\mathrm{T}}$ and

$$
\boldsymbol{\Phi}(\mathbf{x}, \mathbf{y}) \triangleq \frac{2}{\sigma_{w}^{2}} \Re\left\{\frac{\partial \boldsymbol{\mu}^{\mathrm{H}}}{\partial \mathbf{x}} \frac{\partial \boldsymbol{\mu}}{\partial \mathbf{y}^{\mathrm{T}}}\right\}
$$

where

$$
\boldsymbol{\mu}=\overline{\boldsymbol{\Gamma}}(\mathbf{t})\left(\mathbf{I}_{N_{a}} \otimes \mathbf{B}\right) \overline{\mathbf{F}}_{L} \mathbf{h},
$$

with $\overline{\boldsymbol{\Gamma}}(\mathbf{t})=\operatorname{diag}\left\{\overline{\boldsymbol{\Gamma}}\left(\mathbf{t}_{k}\right)\right\}_{k \in \mathcal{N}_{a}}, \quad \mathbf{B}=\operatorname{diag}\left\{\mathbf{B}_{j}\right\}_{j \in \mathcal{N}_{b}}$, $\overline{\mathbf{F}}_{L}=\operatorname{diag}\left\{\overline{\mathbf{F}}_{L_{k}}\right\}_{k \in \mathcal{N}_{a}}, \mathbf{h}=\left[\mathbf{h}_{1}^{\mathrm{T}}, \ldots, \mathbf{h}_{N_{a}}^{\mathrm{T}}\right]^{\mathrm{T}}, \mathbf{w}=$ $\left[\mathbf{w}_{1}^{\mathrm{T}}, \ldots, \mathbf{w}_{N_{a}}^{\mathrm{T}}\right]^{\mathrm{T}}$, in which $\overline{\boldsymbol{\Gamma}}\left(\mathbf{t}_{k}\right), \overline{\mathbf{F}}_{L_{k}}$, and $\mathbf{h}_{k}$ are defined as $\overline{\boldsymbol{\Gamma}}\left(\mathbf{t}_{k}\right)=\operatorname{diag}\left\{\boldsymbol{\Gamma}\left(t_{k, j}\right)\right\}_{j \in \mathcal{N}_{b}}, \overline{\mathbf{F}}_{L_{k}}=\operatorname{diag}\left\{\mathbf{F}_{L_{k, j}}\right\}_{j \in \mathcal{N}_{b}}$, and $\mathbf{h}_{k}=\left[\begin{array}{lll}\mathbf{h}_{k, N_{a}+1}^{\mathrm{T}} & \cdots & \mathbf{h}_{k, N_{a}+N_{b}}^{\mathrm{T}}\end{array}\right]^{\mathrm{T}}$. In addition, $\mathbf{w}_{k}=$ $\left[\begin{array}{llll}\mathbf{w}_{k, N_{a}+1}^{\mathrm{T}} & \cdots & \mathbf{w}_{k, N_{a}+N_{b}}^{\mathrm{T}}\end{array}\right]^{\mathrm{T}}$.

The terms $\boldsymbol{\Phi}\left(\boldsymbol{\theta}_{k}, \boldsymbol{\theta}_{m}\right)$ for $k \neq m$ in the FIM are zero, so that

$$
\mathbf{J}_{\boldsymbol{\eta}}=\left[\begin{array}{cccc}
\mathbf{\Phi}(\mathbf{q}, \mathbf{q}) & \boldsymbol{\Phi}\left(\mathbf{q}, \boldsymbol{\theta}_{1}\right) & \ldots & \boldsymbol{\Phi}\left(\mathbf{q}, \boldsymbol{\theta}_{N_{a}}\right) \\
\mathbf{\Phi}\left(\boldsymbol{\theta}_{1}, \mathbf{q}\right) & \boldsymbol{\Phi}\left(\boldsymbol{\theta}_{1}, \boldsymbol{\theta}_{1}\right) & \ldots & \mathbf{0} \\
\vdots & & \ddots & \vdots \\
\mathbf{\Phi}\left(\boldsymbol{\theta}_{N_{a}}, \mathbf{q}\right) & \mathbf{0} & \ldots & \boldsymbol{\Phi}\left(\boldsymbol{\theta}_{N_{a}}, \boldsymbol{\theta}_{N_{a}}\right)
\end{array}\right]
$$

Using the Schur complement form, we obtain the equivalent FIM (EFIM) of the positions as

$$
\mathbf{J}_{e}(\mathbf{q})=\boldsymbol{\Phi}(\mathbf{q}, \mathbf{q})-\sum_{k=1}^{N_{a}} \mathbf{\Upsilon}\left(\mathbf{q}, \boldsymbol{\theta}_{k}, \mathbf{q}\right),
$$

in which $\boldsymbol{\Upsilon}\left(\mathbf{q}, \boldsymbol{\theta}_{k}, \mathbf{q}\right)=\boldsymbol{\Phi}\left(\mathbf{q}, \boldsymbol{\theta}_{k}\right) \boldsymbol{\Phi}^{-1}\left(\boldsymbol{\theta}_{k}, \boldsymbol{\theta}_{k}\right) \boldsymbol{\Phi}\left(\boldsymbol{\theta}_{k}, \mathbf{q}\right)$. Since the terms $\boldsymbol{\Phi}\left(\mathbf{q}_{k}, \mathbf{q}_{m}\right)$ and $\boldsymbol{\Phi}\left(\mathbf{q}_{k}, \boldsymbol{\theta}_{m}\right)$ are zero for $k \neq m$, the first element of the FIM, $\boldsymbol{\Phi}(\mathbf{q}, \mathbf{q})$, and off-diagonal entries of the form, $\boldsymbol{\Phi}\left(\mathbf{q}_{k}, \boldsymbol{\theta}_{k}\right)$, are block-diagonal matrices. Hence, we obtain the EFIM of the agent positions $\mathbf{J}_{e}(\mathbf{q})$ as

$$
\begin{aligned}
& \mathbf{J}_{e}(\mathbf{q})= \\
& \frac{2}{c^{2} \sigma_{w}^{2}} \sum_{j \in \mathcal{N}_{b}} \operatorname{diag}\left\{\lambda_{1, j} \mathbf{J}_{r}\left(\phi_{1, j}\right), \ldots, \lambda_{N_{a}, j} \mathbf{J}_{r}\left(\phi_{N_{a}, j}\right)\right\},
\end{aligned}
$$


where we have introduced the ranging direction matrix $(\mathrm{RDM}) \mathbf{J}_{r}\left(\phi_{k, j}\right)=\mathbf{u}_{r}\left(\phi_{k, j}\right) \mathbf{u}_{r}^{\mathrm{T}}\left(\phi_{k, j}\right)$ with $\mathbf{u}_{r}\left(\phi_{k, j}\right)=$ $\left[\cos \phi_{k, j} \sin \phi_{k, j}\right]^{\mathrm{T}}$, in which $\phi_{k, j}= \pm \arctan \left(y_{k}-y_{j}\right) /\left(x_{k}-\right.$ $x_{j}$ ) represents the angle between the $j$ th anchor and $k$ th agent (with positive sign for $x_{k}>x_{j}$ and $y_{k}>y_{j}$ or $x_{k}<x_{j}$ and $y_{k}<y_{j}$ and negative sign otherwise). The ranging information intensity (RII) $\lambda_{k, j}$ can be decomposed as information for the channel $\mathbf{h}_{k, j}$ and a reduction due to the uncertainty of the channel $\mathbf{h}_{k, j}$ (see Appendix A for a complete derivation):

$$
\lambda_{k, j}=\mathbf{h}_{k, j}^{\mathrm{H}} \mathbf{M}_{k, j}^{(1)} \mathbf{h}_{k, j}-\mathbf{h}_{k, j}^{\mathrm{H}} \mathbf{M}_{k, j}^{(2)} \mathbf{h}_{k, j},
$$

where

$$
\mathbf{M}_{k, j}^{(1)}=\mathbf{F}_{L_{k, j}}^{\mathrm{H}} \mathbf{D}^{\mathrm{H}} \mathbf{P}_{j} \mathbf{D} \mathbf{F}_{L_{k, j}},
$$

in which $\mathbf{D}=\operatorname{diag}\{j 2 \pi n / T\}_{n=0}^{N-1}$ and

$$
\mathbf{M}_{k, j}^{(2)}=\boldsymbol{\Xi}_{k, j}^{\mathrm{H}} \boldsymbol{\Sigma}_{k, j}^{-1} \boldsymbol{\Xi}_{k, j},
$$

with $\boldsymbol{\Xi}_{k, j}=\mathbf{F}_{L_{k, j}}^{\mathrm{H}} \mathbf{P}_{j} \mathbf{D} \mathbf{F}_{L_{k, j}}$ and $\boldsymbol{\Sigma}_{k, j}=\mathbf{F}_{L_{k, j}}^{\mathrm{H}} \mathbf{P}_{j} \mathbf{F}_{L_{k, j}}$.

\section{B. SPEB for a Specific Channel and Agent Location}

Using the fact that the EFIM of agent positions is of blockdiagonal form presented in (10), we define the SPEB for $k$ th agent as

$$
\mathcal{P}_{k}(\mathbf{p}) \triangleq \operatorname{tr}\left\{\mathbf{J}_{e}^{-1}\left(\mathbf{q}_{k}\right)\right\}
$$

where

$$
\mathbf{J}_{e}\left(\mathbf{q}_{k}\right)=\frac{2}{c^{2} \sigma_{w}^{2}} \sum_{j \in \mathcal{N}_{b}} \lambda_{k, j} \mathbf{J}_{r}\left(\phi_{k, j}\right) .
$$

Considering (11) and (14), it can be observed that SPEB for the agents is a function of input power $\mathbf{p}$, the relative angles between the agents and anchors $\phi_{k, j}$, and channel coefficients $\mathbf{h}_{k, j}$.

\section{SPEB for a Set of Channels and Agent Locations}

The SPEB in (14) cannot be used directly as a constraint for power allocation, since it presumes knowledge of the agents' positions, which are precisely the parameters to be estimated. In contrast, having no knowledge at all about the positions of the agents would mean that we cannot perform any intelligent power allocation. In this section, we consider a middle ground where have side information regarding the channels and agents' positions. This side information can be in the form of a set or, more generally, a distribution. Including this side information in (14) will allow us to perform robust power allocation.

The expected SPEB is defined as

$$
\overline{\mathcal{P}}_{k}(\mathbf{p}) \triangleq \operatorname{tr}\left\{\mathbb{E}_{\boldsymbol{\eta}}\left[\mathbf{J}_{e}^{-1}\left(\mathbf{q}_{k}\right)\right]\right\},
$$

where the expectation is carried out over the distribution of the channel coefficients $\mathbf{h}_{k, j}$ and agents' positions $\mathbf{q}_{k}$. To evaluate expected SPEB we consider a scenario in which the agents were previously located at certain known positions (with certain $\phi_{k, j}^{(-)}$) and with certain known channel coefficients $\mathbf{h}_{k, j}^{(-)}$. Then, the agent moves, giving rise to a set of possible channel coefficients

$$
\mathbf{h}_{k, j}^{(+)}=\mathbf{h}_{k, j}^{(-)}+\Delta \mathbf{h}_{k, j}
$$

and relative angles

$$
\phi_{k, j}^{(+)}=\phi_{k, j}^{(-)}+\Delta \phi_{k, j},
$$

with $\boldsymbol{\Delta} \mathbf{h}_{k, j}$ and $\Delta \phi_{k, j}$ drawn from appropriate distributions that capture the uncertainty regarding the channels and positions after movement.

We can approximate the expected SPEB using numerical averaging over $M_{q}$ realizations:

$$
\mathcal{P}_{k}(\mathbf{p}) \approx \overline{\mathcal{P}}_{k, \text { ap }}(\mathbf{p}),
$$

where

$\overline{\mathcal{P}}_{k, \text { ap }}(\mathbf{p})=\frac{1}{M_{q}} \sum_{m=1}^{M_{q}} \operatorname{tr}\left\{[\underbrace{\frac{2}{c^{2} \sigma_{w}^{2}} \sum_{j \in \mathcal{N}_{b}} \lambda_{k, j, m}^{(+)} \mathbf{J}_{r}\left(\phi_{k, j, m}^{(+)}\right)}_{\mathbf{J}_{k, m}}]^{-1}\right\}$

where $\mathbf{M}_{k, j}^{(1)}$ and $\mathbf{M}_{k, j}^{(2)}$ were defined in (12)-(13),

$$
\lambda_{k, j, m}^{(+)}=\left(\mathbf{h}_{k, j, m}^{(+)}\right)^{\mathrm{H}}\left(\mathbf{M}_{k, j}^{(1)}-\mathbf{M}_{k, j}^{(2)}\right) \mathbf{h}_{k, j, m}^{(+)},
$$

with $\mathbf{h}_{k, j, m}^{(+)}$and $\phi_{k, j, m}^{(+)}$being generated as

$$
\begin{aligned}
\mathbf{h}_{k, j, m}^{(+)} & =\mathbf{h}_{k, j}^{(-)}+\Delta \mathbf{h}_{k, j, m} \\
\phi_{k, j, m}^{(+)} & =\phi_{k, j}^{(-)}+\Delta \phi_{k, j, m},
\end{aligned}
$$

in which $\boldsymbol{\Delta} \mathbf{h}_{k, j, m}$ and $\Delta \phi_{k, j, m}$ denote the $m$ th sample from the distributions of $\boldsymbol{\Delta} \mathbf{h}_{k, j}$ and $\Delta \phi_{k, j}$, respectively.

\section{Robust Power Allocation}

We can now formulate an optimization problem to minimize the total power required to achieve a certain positioning accuracy on the agents' positions, considering the uncertainty on the channel coefficients and relative angles between anchors and agents. First, we propose the general problem formulation and explain how multicast design differs from the unicast design. Then, the SDP form of the problem will be presented.

\section{A. Formulation}

The robust problem for the case of multicast design has the following form

$$
\begin{array}{cc}
\underset{\mathbf{p}}{\operatorname{minimize}} & \sum_{j \in \mathcal{N}_{b}} \mathbf{1}^{\mathrm{T}} \mathbf{p}_{j} \\
\text { subject to } & \overline{\mathcal{P}}_{k, \text { ap }}(\mathbf{p}) \leq \beta, \forall k \in \mathcal{N}_{a} \\
& \mathbf{p}_{j} \succeq \mathbf{0}, \forall j \in \mathcal{N}_{b},
\end{array}
$$

where $\beta$ is the desired upper bound on the SPEB of the agents. We note that (24a) aims to minimize the total power of the anchors required to localize the agents, while (24b) sets an 
upper bound $\beta$ to the SPEB of the agents and (24c) restricts the anchor power vectors $\mathbf{p}_{j}$ to be non-negative.

The problem for the case of unicast design is

$$
\begin{array}{cc}
\underset{\mathbf{p}}{\operatorname{minimize}} & \sum_{k \in \mathcal{N}_{a}} \sum_{j \in \mathcal{N}_{b}} \mathbf{1}^{\mathrm{T}} \mathbf{p}_{k, j} \\
\text { subject to } & \overline{\mathcal{P}}_{k, \text { ap }}\left(\mathbf{p}_{k,:}\right) \leq \beta, \forall k \in \mathcal{N}_{a} \\
& \mathbf{p}_{k, j} \succeq \mathbf{0}, \forall j \in \mathcal{N}_{b} ; \forall k \in \mathcal{N}_{a},
\end{array}
$$

which is the multicarrier extension of [8].

\section{B. SDP Reformulation}

Since the robust power allocation problems are not in a standard form, we will reformulate them. We can replace $\mathbf{J}_{k, m}^{-1}$ from (20) with an auxiliary matrix $\widetilde{\mathbf{Y}}_{k, m}$ and formulate the SPEB constraint as

$$
\begin{array}{r}
\frac{1}{M_{q}} \sum_{m=1}^{M_{q}} \operatorname{tr}\left\{\widetilde{\mathbf{Y}}_{k, m}\right\} \leq \beta \\
\mathbf{J}_{k, m}^{-1} \preceq \widetilde{\mathbf{Y}}_{k, m} .
\end{array}
$$

Defining $\mathbf{Y}_{k, m}$ as $\mathbf{Y}_{k, m}=\widetilde{\mathbf{Y}}_{k, m}^{-1}$, we obtain an equivalent form:

$$
\begin{array}{r}
\frac{1}{M_{q}} \sum_{m=1}^{M_{q}} \operatorname{tr}\left\{\mathbf{Y}_{k, m}^{-1}\right\} \leq \beta \\
\mathbf{J}_{k, m} \succeq \mathbf{Y}_{k, m} .
\end{array}
$$

Now, (28) can be written in the SDP form [11]:

$$
\boldsymbol{\Theta}\left(\mathbf{Y}_{k} ; \beta\right) \succeq \mathbf{0}
$$

in which

$$
\boldsymbol{\Theta}\left(\mathbf{Y}_{k} ; \beta\right)=\left[\begin{array}{ccc}
M_{q} \beta & \mathbf{v}_{1}^{\mathrm{T}} & \mathbf{v}_{2}^{\mathrm{T}} \\
\mathbf{v}_{1} & \mathbf{Y}_{k} & \mathbf{0} \\
\mathbf{v}_{2} & \mathbf{0} & \mathbf{Y}_{k}
\end{array}\right]
$$

with $\mathbf{Y}_{k}=\operatorname{diag}\left\{\mathbf{Y}_{k, m}\right\}_{m=1}^{M_{q}}, \mathbf{v}_{1}=\mathbf{1}_{M_{q}} \otimes \widetilde{\mathbf{v}}_{1}$ and $\mathbf{v}_{2}=$ $\mathbf{1}_{M_{q}} \otimes \widetilde{\mathbf{v}}_{2}$ in which $\widetilde{\mathbf{v}}_{1}=[1,0]^{T}$ and $\widetilde{\mathbf{v}}_{2}=[0,1]^{T}$.

A linear matrix inequality (LMI) form of (29) is obtained as

$$
\boldsymbol{\Pi}_{k, m} \succeq \mathbf{0}
$$

where

$$
\begin{aligned}
& \boldsymbol{\Pi}_{k, m}= \\
& {\left[\begin{array}{cc}
\sum_{j \in \mathcal{N}_{b}} \nu_{k, j, m} \mathbf{J}_{r}\left(\phi_{k, j, m}^{(+)}\right)-\mathbf{Y}_{k, m} & \mathbf{U}_{k, m}^{\mathrm{H}} \\
\mathbf{U}_{k, m} & \operatorname{diag}\left\{\boldsymbol{\Sigma}_{k, j}\right\}_{j \in \mathcal{N}_{b}}
\end{array}\right]}
\end{aligned}
$$

in which

$$
\begin{aligned}
\nu_{k, j, m} & =\left(\mathbf{h}_{k, j, m}^{(+)}\right)^{\mathrm{H}} \mathbf{M}_{k, j}^{(1)} \mathbf{h}_{k, j, m}^{(+)}, \\
\mathbf{U}_{k, m}^{\mathrm{H}} & =\left[\begin{array}{lll}
\mathbf{U}_{k, 1, m}^{\mathrm{H}} & \cdots & \mathbf{U}_{k, N_{b}, m}^{\mathrm{H}}
\end{array}\right] \\
\mathbf{U}_{k, j, m}^{\mathrm{H}} & =\mathbf{u}\left(\phi_{k, j, m}^{(+)}\right)\left(\mathbf{h}_{k, j, m}^{(+)}\right)^{\mathrm{H}} \boldsymbol{\Xi}_{k, j}^{\mathrm{H}} .
\end{aligned}
$$

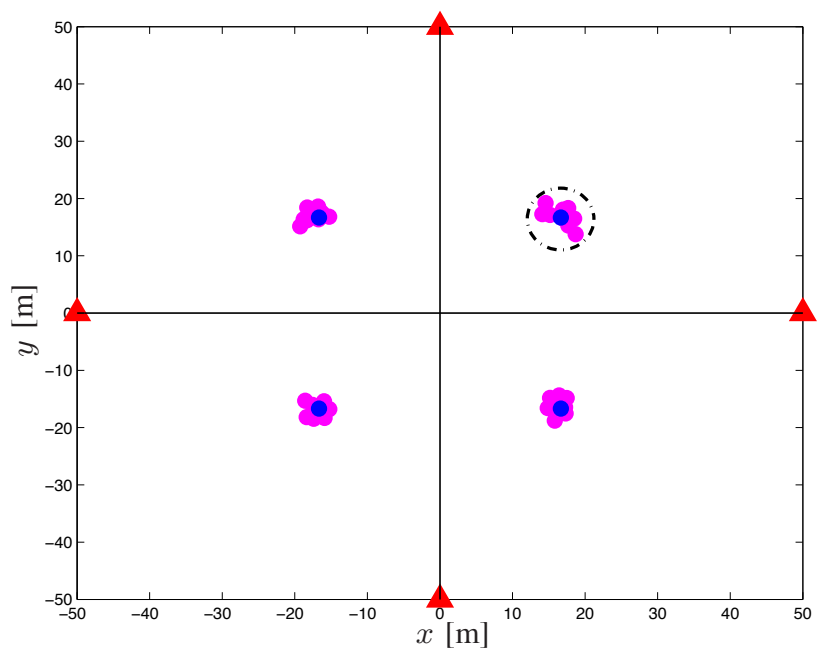

Fig. 1. Network topology with four anchors (red triangles) and four agents (blue circles) over the $[-50 \mathrm{~m}, 50 \mathrm{~m}] \times[-50 \mathrm{~m}, 50 \mathrm{~m}]$ map. The pink discs represent possible positions after movement.

Finally, using (30) and (32) as a replacement for (24b), we obtain

$$
\begin{array}{cc}
\underset{\mathbf{p},\left\{\mathbf{Y}_{k, m}\right\}}{\operatorname{minimize}} & \sum_{j \in \mathcal{N}_{b}} \mathbf{1}^{\mathrm{T}} \mathbf{p}_{j} \\
\text { subject to } & \boldsymbol{\Theta}\left(\mathbf{Y}_{k} ; \beta\right) \succeq \mathbf{0}, \forall k \in \mathcal{N}_{a} \\
& \mathbf{\Pi}_{k, m} \succeq \mathbf{0}, \forall k \in \mathcal{N}_{a}, \forall m \in \mathcal{M}_{q} \\
& \mathbf{p}_{j} \succeq \mathbf{0}, \forall j \in \mathcal{N}_{b},
\end{array}
$$

where $\mathcal{M}_{q}=\left\{1, \ldots, M_{q}\right\}$. The expressions in (37a)-(37d) describe a convex optimization problem of SDP form. Similarly, the SDP form of unicast design is obtained by replacing $\mathbf{p}_{j}$ by $\mathbf{p}_{k, j}$ and the cost function as $\sum_{k \in \mathcal{N}_{a}} \sum_{j \in \mathcal{N}_{b}} \mathbf{1}^{\mathrm{T}} \mathbf{p}_{k, j}$.

\section{Simulation Results}

In this section, we analyze our robust design in terms of the total power and achieved SPEB, for both multicast and unicast methods. We compare the robust design with a nonrobust design.

\section{A. Simulation Setup}

We consider networks with $N_{b}=4$ fixed anchors and $N_{a}=4$ agents with varying positions in a square area of $100 \mathrm{~m} \times 100 \mathrm{~m}$ as shown in Fig. 1, for a given bandwidth of $N / T=480 \mathrm{kHz}, N=32$ subcarriers, and a noise power of $\sigma_{w}^{2}=0.1$. We set $\beta=0.5 \mathrm{~m}^{2}$ (i.e., a target uncertainty or around $70 \mathrm{~cm}$ ). We assume that the uncertainty in the channels and agents' positions originates from mobility. In particular, before moving, agents are in the locations $\mathbf{q}^{(-)}$, indicated by blue dots in Fig. 1. After mobility, they may move in any direction (pink dots) and cross a distance up to $10 \mathrm{~m}$ (indicated by the dashed circle in Fig. 1). The new locations are denoted by $\mathbf{q}^{(+)}$and are unknown during power allocation. We will model the distribution of $\Delta \phi_{k, j}$ as uniform in an interval $\left[-\Delta_{k, j},+\Delta_{k, j}\right]$, derived from the geometry of the scenario. 


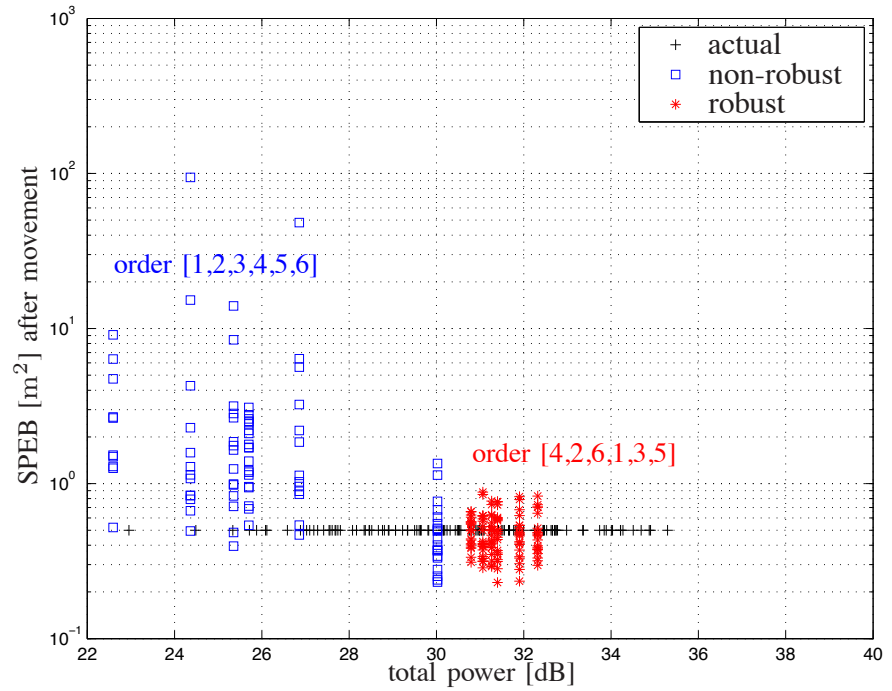

Fig. 2. Multicast: SPEB after movement vs total allocated power for 6 network realization and 20 channel and position values after movement, for 3 power allocations: actual, robust, and non-robust.

In terms of the channel coefficients, $L=5$ tap channels $\mathbf{h}_{k, j}^{(-)}$ before movement are generated as $\mathbf{h}_{k, j}^{(-)}=\sqrt{5} \mathbf{g}_{k, j} /\left\|\mathbf{g}_{k, j}\right\|$, in which $\mathbf{g}_{k, j} \sim \mathcal{C N}\left(\mathbf{0}, \mathbf{I}_{L}\right)$. Channel uncertainty is modeled through $\boldsymbol{\Delta} \mathbf{h}_{k, j} \sim \mathcal{C N}\left(\mathbf{0}, 0.1 \mathbf{I}_{L}\right)$. We will consider 6 different realizations of the network (i.e., channels and positions). For each realization, we consider 20 possible channel and position values after movement to evaluate the robustness.

We have considered three distinct power allocation approaches, for both unicast and multicast:

- Actual: Power allocation is based on actual positions and channels. As a (non-causal) benchmark, we determine the optimal power allocation given $\mathbf{h}_{k, j}^{(+)}$and $\mathbf{q}_{k}^{(+)}, \forall k, j$. This is achieved by solving the SDP using (14) as an SPEB constraint.

- Robust: Power allocation is found by solving the SDP using the average SPEB constraint with $M_{q}=20$, given $\mathbf{h}_{k, j}^{(-)}$and $\mathbf{q}_{k}^{(-)}$as well as the $M_{q}$ possible channel and position values after movement.

- Non-robust: Power allocation is found by solving the SDP using (14) as an SPEB constraint, wherein we use the values $\mathbf{h}_{k, j}^{(-)}$and $\mathbf{q}_{k}^{(-)}$. Hence, the power allocation is based on outdated channel and position information.

For each of these three approaches, we determine the total power as well as the actual SPEB under the power allocation, for each of the 20 possible channel and position values after movement. The SDPs and convex programs are solved using CVX [12].

\section{B. Results and Discussion}

The results for multicast and unicast are shown in Fig. 2 and Fig. 3, respectively. In each figure, we show the realized SDEP vs total power for the three approaches (actual in black plus signs, robust in red stars, and non-robust in blue squares). As we considered 6 network realizations, and $M_{q}=20$ for each

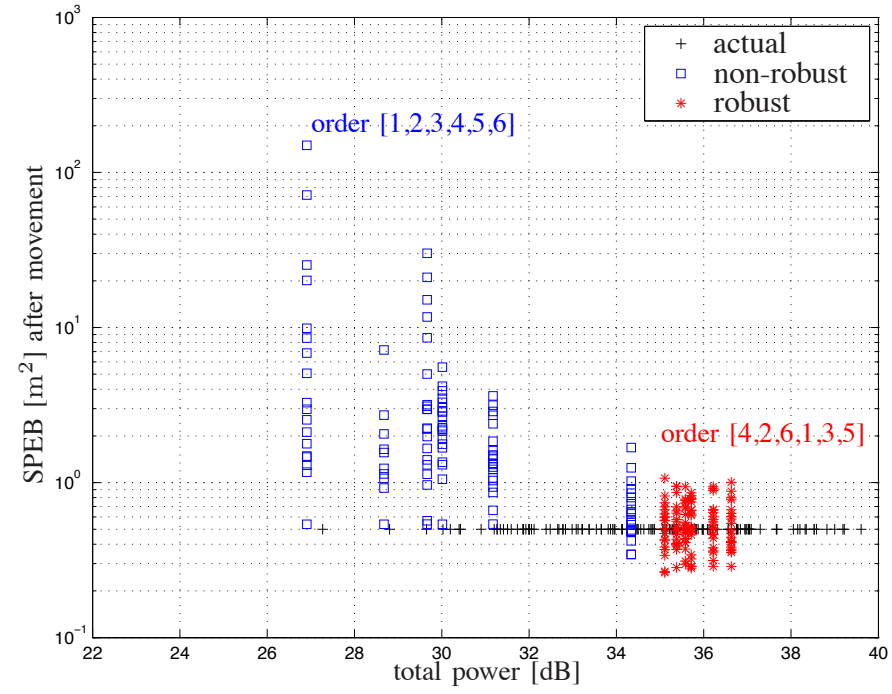

Fig. 3. Unicast: SPEB after movement vs total allocated power for 6 network realization and 20 channel and position values after movement, for 3 power allocations: actual, robust, and non-robust.

network realization, there are 120 markers for each approach. For the robust and non-robust allocations, the power does not depend on the channel or position after movement, which leads to clustering of the markers in columns, one for each network realization. Note, however, that the realized SPEB after movement does depend on the channel and position after movement, thus leading to a spread in each column for the given power allocation. For the design based on the actual channels and positions after movement, there is no obvious clustering, so we did not separate results for the individual network realizations.

We first consider Fig. 2. We see that the allocation based on the actual channels and positions leads to a constant SPEB of $\beta$, as expected. The non-robust allocation is based solely on the values $\mathbf{h}_{k, j}^{(-)}$and $\mathbf{q}_{k}^{(-)}$and leads to large violations of the SPEB requirement. There is also a large spread in the required power (ranging from $22.5 \mathrm{~dB}$ to $30 \mathrm{~dB}$ ). In the robust power allocation, the total power is larger, but lies within a more narrow range of $30.7 \mathrm{~dB}$ to $32.5 \mathrm{~dB}$. Importantly, the violations of the SPEB requirement are less severe, with SPEB values clustered closely around the target value of 0.5 . We note that the increase in power changes from channel to channel, which is indicated by the order of the network realization for non-robust and robust power allocation: for instance, for the first network realization, the total power for the non-robust allocation was $22.6 \mathrm{~dB}$, while for the robust allocation it was $31.4 \mathrm{~dB}$ (i.e., an increase of $8.8 \mathrm{~dB}$ ), while for the sixth network, the increase is only from $30 \mathrm{~dB}$ to $31.3 \mathrm{~dB}$ (i.e., an increase of $1.3 \mathrm{~dB}$ ). We note also that the total power of the robust allocation will depend on the uncertainty in the channel and position after movement, with larger uncertainties leading to more total power allocated to satisfy the expected SPEB constraint.

For unicast, Fig. 3 indicates similar trends. We observe 
that unicast requires more power than multicast, as unicast requires more transmissions, i.e., unicast requires four times more transmissions. However, unicast does not need four times the power of multicast, as the power allocation can be tailored better to the characteristics of the individual channels and user positions.

\section{CONCLUSION}

We have investigated robust power allocation for unicast and multicast OFDM-based positioning with an accuracy constraint. The robustness to uncertainties is addressed through an expected SPEB constraint. The expected SPEB constraint incorporates statistical knowledge of expected channels and positions, and leads to reduced violations of the accuracy requirements compared to a non-robust power allocation, at a cost of an increase in total power.

\section{ACKNOWLEDGMENT}

This work was supported by the European Commission, under EU FP7 Marie Curie Initial Training Network MULTIPOS (Multi-technology Positioning Professionals) under grant nr. 316528, and the European Research Council, under grant nr. 258418 (COOPNET).

\section{APPENDIX A \\ DETAILS OF (11)}

The $k$ th block of the first term $\boldsymbol{\Phi}(\mathbf{q}, \mathbf{q})$ can be obtained by the chain rule for the derivation as

$$
\begin{aligned}
\boldsymbol{\Phi}\left(\mathbf{q}_{k}, \mathbf{q}_{k}\right) & =\frac{\partial \boldsymbol{\mu}_{k, j}^{\mathrm{H}}}{\partial \mathbf{q}_{k}} \frac{\partial \boldsymbol{\mu}_{k, j}}{\partial \mathbf{q}_{k}^{\mathrm{T}}} \\
& =\frac{\partial d_{k, j}}{\partial \mathbf{q}_{k}} \frac{\partial \boldsymbol{\mu}_{k, j}^{\mathrm{H}}}{\partial d_{k, j}} \frac{\partial \boldsymbol{\mu}_{k, j}}{\partial d_{k, j}} \frac{\partial d_{k, j}}{\partial \mathbf{q}_{k}^{\mathrm{T}}},
\end{aligned}
$$

where $\boldsymbol{\mu}_{k, j}=\boldsymbol{\Gamma}\left(t_{k, j}\right) \mathbf{B}_{j} \mathbf{F}_{L_{k, j}} \mathbf{h}_{k, j}$. Consequently, we obtain

$$
\boldsymbol{\Phi}\left(\mathbf{q}_{k}, \mathbf{q}_{k}\right)=\frac{1}{c^{2}} \sum_{j \in \mathcal{N}_{b}} \mathbf{h}_{k, j}^{\mathrm{H}} \mathbf{M}_{k, j}^{(1)} \mathbf{h}_{k, j} \mathbf{J}_{r}\left(\phi_{k, j}\right) .
$$

The $k$ th block of $\boldsymbol{\Phi}\left(\mathbf{q}, \boldsymbol{\theta}_{k}\right)$ forms a block-diagonal matrix with the $j$ th entry $\boldsymbol{\Phi}_{k, j}\left(\mathbf{q}_{k}, \mathbf{k}_{k, j}\right)$ defined as

$$
\boldsymbol{\Phi}_{k, j}\left(\mathbf{q}_{k}, \mathbf{k}_{k, j}\right)=\frac{\partial \boldsymbol{\mu}_{k, j}^{\mathrm{H}}}{\partial \mathbf{q}_{k}} \frac{\partial \boldsymbol{\mu}_{k, j}}{\partial \mathbf{k}_{k, j}^{\mathrm{T}}}=\frac{\partial d_{k, j}}{\partial \mathbf{q}_{k}} \frac{\partial \boldsymbol{\mu}_{k, j}^{\mathrm{H}}}{\partial d_{k, j}} \frac{\partial \boldsymbol{\mu}_{k, j}}{\partial \mathbf{k}_{k, j}^{\mathrm{T}}} .
$$

Consequently, we obtain

$$
\boldsymbol{\Phi}_{k, j}\left(\mathbf{q}_{k}, \mathbf{k}_{k, j}\right)=\frac{1}{c} \mathbf{u}_{r}\left(\phi_{k, j}\right)\left[\Re\left\{\boldsymbol{\rho}_{k, j}^{\mathrm{H}}\right\} \quad \Im\left\{\boldsymbol{\rho}_{k, j}^{\mathrm{H}}\right\}\right],
$$

where $\boldsymbol{\rho}_{k, j}=\boldsymbol{\Xi}_{k, j} \mathbf{h}_{k, j}$. The $j$ th element of the block-diagonal matrix $\boldsymbol{\Phi}\left(\boldsymbol{\theta}_{k}, \boldsymbol{\theta}_{k}\right)$ is obtained as

$$
\frac{\partial \boldsymbol{\mu}_{k, j}^{\mathrm{H}}}{\partial \mathbf{k}_{k, j}} \frac{\partial \boldsymbol{\mu}_{k, j}}{\partial \mathbf{k}_{k, j}^{\mathrm{T}}}=\left[\begin{array}{cc}
\Re\left\{\boldsymbol{\Sigma}_{k, j}\right\} & -\Im\left\{\boldsymbol{\Sigma}_{k, j}\right\} \\
\Im\left\{\boldsymbol{\Sigma}_{k, j}\right\} & \Re\left\{\boldsymbol{\Sigma}_{k, j}\right\}
\end{array}\right] .
$$

Finally, substituting (40), (42), and (43) in (9), the corresponding $\lambda_{k, j}$ in (10) can be found as (11).

\section{REFERENCES}

[1] A. H. Sayed, A. Tarighat, and N. Khajehnouri, "Network-based wireless location: Challenges faced in developing techniques for accurate wireless location information," IEEE Signal Process. Mag., vol. 22, no. 4, pp. 24-40, Jul. 2005.

[2] H. Godrich, A. M. Haimovich, and R. S. Blum, "Target localization accuracy gain in MIMO radar-based systems," IEEE Trans. Inf. Theory, vol. 56, no. 6, pp. 2783-2803, Jul. 2005.

[3] F. Meshkati, H. V. Poor, and S. C. Schwartz, "Energy-efficient resource allocation in wireless networks," IEEE Signal Process. Mag., vol. 24, no. 3, pp. 58-68, May. 2007.

[4] Y. Shen, W. Dai, and M. Z. Win, "Power optimization for network localization," IEEE/ACM Trans. Netw., vol. 22, no. 4, pp. 1337-1350, Aug. 2014.

[5] W. Dai, S. Yuan, and M. Z. Win, "Distributed power allocation for cooperative wireless network localization," IEEE Journal on Selected Areas in Communications, Feb. 2014.

[6] Y. Shen and M. Z. Win, "Fundamental limits of wideband localizationpart I: A general framework," IEEE Trans. Inf. Theory, vol. 56, no. 10, pp. 4956-4980, Oct. 2010.

[7] Y. Shen, H. Wymeersch, and M. Z. Win, "Fundamental limits of wideband localization part II: Cooperative networks," IEEE Trans. Inf. Theory, vol. 56, no. 10, pp. 4981-4999, Oct. 2010.

[8] W. W. L. Li, Y. Shen, Y. J. Zhang, and M. Z. Win, "Robust power allocation for energy-efficient location-aware networks," IEEE/ACM Trans. Netw, vol. 21, no. 6, pp. 1918-1930, Dec. 2013.

[9] M. Larsen, G. Seco-Granados, and A. Swindlehurst, "Pilot optimization for time-delay and channel estimation in OFDM systems," in IEEE Int. Conf. on Acoustics, Speech and Signal Processing (ICASSP), 2011, pp. 3564-3567.

[10] S. M. Kay, Fundamentals of Statistical Signal Processing: Estimation Theory. New York, NY, USA: Prentice Hall, 2010.

[11] S. Boyd and L. Vandenberghe, Convex Optimization. New York, NY, USA: Cambridge University Press, 2004.

[12] M. Grant and S. Boyd, CVX: Matlab software for disciplined convex programming, version 1.21, http://cvxr.com/cvx, Aug. 2010. 